\title{
A Dual-Modality Photoacoustic and Ultrasound Imaging System for Noninvasive Sentinel Lymph Node Detection: Preliminary Clinical Results
}

\author{
Todd N. Erpelding ${ }^{1}$, Alejandro Garcia-Uribe ${ }^{2}$, Arie Krumholz ${ }^{2}$, Haixin $\mathrm{Ke}^{2}$, \\ Konstantin Maslov², Catherine Appleton ${ }^{3}$, Julie Margenthaler ${ }^{3}$, Lihong V. Wang ${ }^{2}$ \\ ${ }^{1}$ Philips Research North America, Briarcliff Manor, NY, USA \\ ${ }^{2}$ Department of Biomedical Engineering, Washington University in St. Louis, MO, USA \\ ${ }^{3}$ Washington University School of Medicine, St. Louis, MO, USA
}

\begin{abstract}
Sentinel lymph node biopsy (SLNB) has emerged as an accurate, less invasive alternative to axillary lymph node dissection, and it has rapidly become the standard of care for patients with clinically node-negative breast cancer. The sentinel lymph node (SLN) hypothesis states that the pathological status of the axilla can be accurately predicted by determining the status of the first (i.e., sentinel) lymph nodes that drain from the primary tumor. Physicians use radio-labeled sulfur colloid and/or methylene blue dye to identify the SLN, which is most likely to contain metastatic cancer cells. However, the surgical procedure causes morbidity and associated expenses. To overcome these limitations, we developed a dual-modality photoacoustic and ultrasound imaging system to noninvasively detect SLNs based on the accumulation of methylene blue dye. Ultimately, we aim to guide percutaneous needle biopsies and provide a minimally invasive method for axillary staging of breast cancer. The system consists of a tunable dye laser pumped by a Nd:YAG laser, a commercial ultrasound imaging system (Philips iU22), and a multichannel data acquisition system which displays co-registered photoacoustic and ultrasound images in real-time. Our clinical results demonstrate that real-time photoacoustic imaging can provide sensitive and specific detection of methylene blue dye in vivo. While preliminary studies have shown that in vivo detection of SLNs by using co-registered photoacoustic and ultrasound imaging is feasible, further investigation is needed to demonstrate robust SLN detection.
\end{abstract}

Keywords: Photoacoustic imaging, ultrasound imaging, sentinel lymph node biopsy, methylene blue, breast cancer.

\section{INTRODUCTION}

The detection of regional lymph node metastases is important in cancer staging as it influences the prognosis of the patient and the strategy for treatment. Sentinel lymph node biopsy (SLNB) has emerged as the standard of care for axillary staging of clinically node-negative breast cancer [1,2]. The sentinel node hypothesis states that the histopathologic status of the first (i.e., sentinel) node on the lymphatic drainage pathway from a primary tumor reflects the

Photons Plus Ultrasound: Imaging and Sensing 2014, edited by Alexander A. Oraevsky, Lihong V. Wang, Proc. of SPIE Vol. 8943, 894359 - (C) 2014 SPIE · CCC code: 1605-7422/14/\$18 - doi: 10.1117/12.2040475 
pathological status of the axilla. Besides the presence of metastasis or micrometastasis detected in the sentinel lymph node (SLN) after excision and histological examination, the total number of involved regional lymph nodes is important in staging the disease, with the number predicting overall survival with an inverse relationship [3].

Typically, the conventional SLNB procedure consists of injecting radioactive tracers and/or methylene blue dye to mark the lymphatic system and guide the surgeon to the sentinel node. The radioactive tracer is injected a few hours prior to the surgery, while methylene blue, which spreads relatively quickly through lymph vessels, is injected in the operating room. A few minutes following methylene blue injection, a surgical incision is made in the area indicated by a hand-held Geiger counter. The surgeon interrogates the axilla and identifies nodes that have been stained blue or nodes that are detected as radioactive with the Geiger counter. These nodes are then removed for histological examination to determine the presence of tumor metastases.

Here, we propose the use of dual-modality photoacoustic tomography (PAT) [4-7] and ultrasonography (US) for accurate identification of SLNs. The US component of PAT-US enables the anatomical identification of lymph nodes, while the PAT component determines if the lymph node is a SLN by imaging accumulated blue dye. Unlike US, PAT is very sensitive to the strong optical absorption of the blue dye. PAT also has higher spatial resolution in deep tissues than pure optical imaging modalities. An accurate identification of SLN by PAT-US can allow sampling of the SLN using fine needle aspiration biopsy (FNAB).

\section{MATERIALS AND METHODS}

\subsection{Photoacoustic and ultrasound imaging system}

We developed a photoacoustic tomography (PAT) and ultrasound (US) imaging system around a modified clinical US scanner (iU22, Philips Healthcare). A system diagram is shown in Fig. 1. The light source consisted of a wavelength tunable dye laser (PrecisionScan-P, Sirah, Kaarst, Germany) pumped by a Q-switched Nd:YAG laser (QuantaRay PRO-350-10, SpectraPhysics, Santa Clara, CA). The laser pulse was coupled to a fused end, bifurcated fiber bundle that flanked both sides of commercially available Philips transducers (L12-5 and L8-4, Philips), (Fig. 2). The laser emitted pulses at a repetition rate of $10 \mathrm{~Hz}$ and pulse duration of $6.5 \mathrm{~ns}$. An optical wavelength near the peak absorption wavelength of methylene blue dye $(667 \mathrm{~nm})$ was chosen, and light fluence on the skin was less than $10 \mathrm{~mJ} / \mathrm{cm}^{2}$, which was within the ANSI safety limits by a factor of 2 [8]. All per-channel data from the US transducer were transferred to a custom-built data acquisition (DAQ) computer, which performed image reconstruction and displayed PA and US and co-registered images at 5 frames/s.

\subsection{Clinical study}

First, a periareolar injection of methylene blue dye was administered as a PAT contrast agent to highlight the SLN. Since methylene blue is commonly used clinically for SLN biopsy, the PAT imaging method builds on the proven track record and physician experience with the dye for SLN detection. Methylene blue dye drains into the sentinel node within minutes of injection, although lymphatic flow can be accelerated using breast massage. Next, axillary PAT-US 
imaging is performed. US imaging detects lymph nodes based on their characteristic appearance (hypoechoic outer cortex and hyperechoic central hilum), while PAT distinguishes sentinel nodes from downstream nodes based on strong photoacoustic contrast from methylene blue.

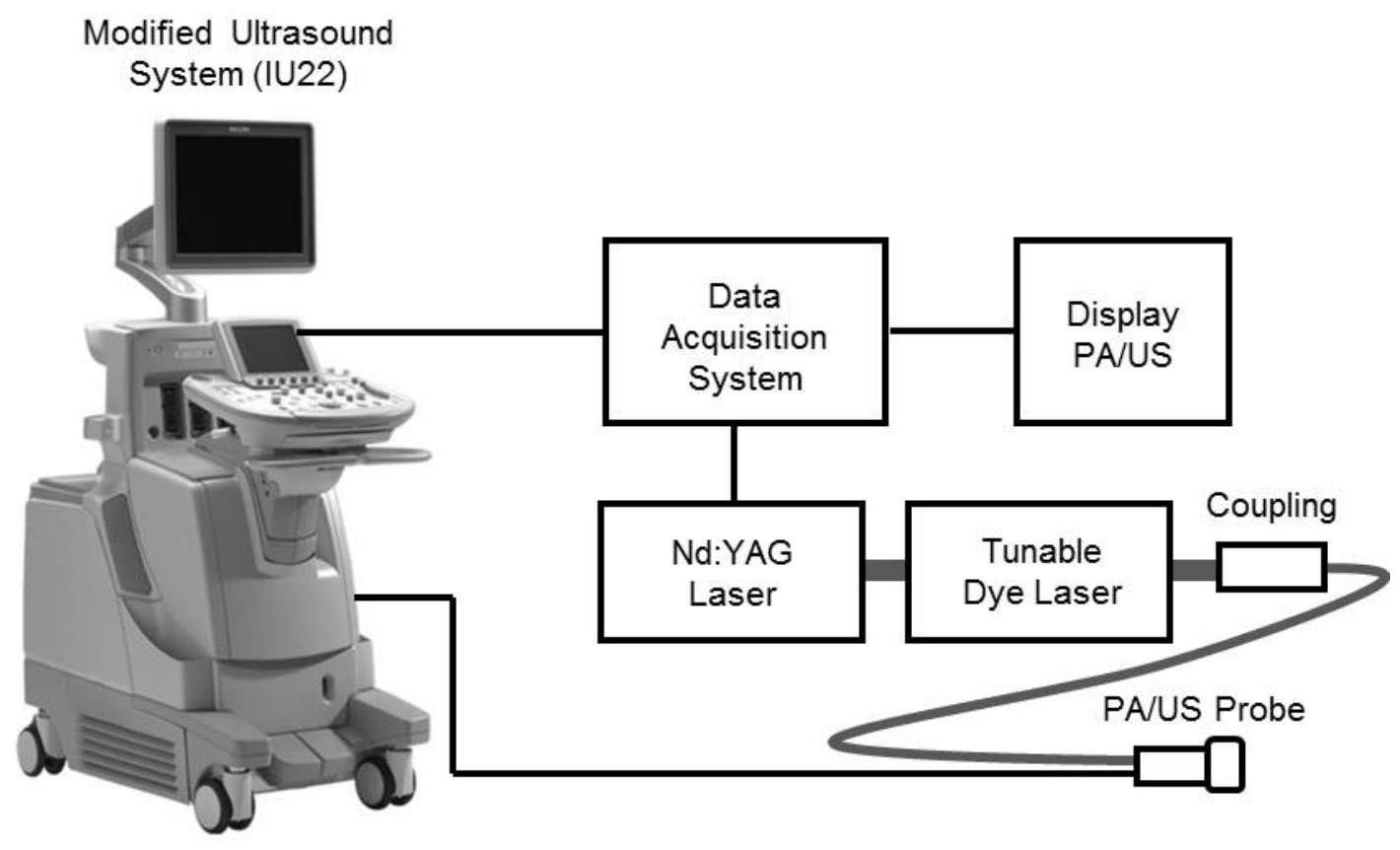

Figure 1. Schematic of the dual-modality photoacoustic and ultrasound imaging system and improved transducer design.

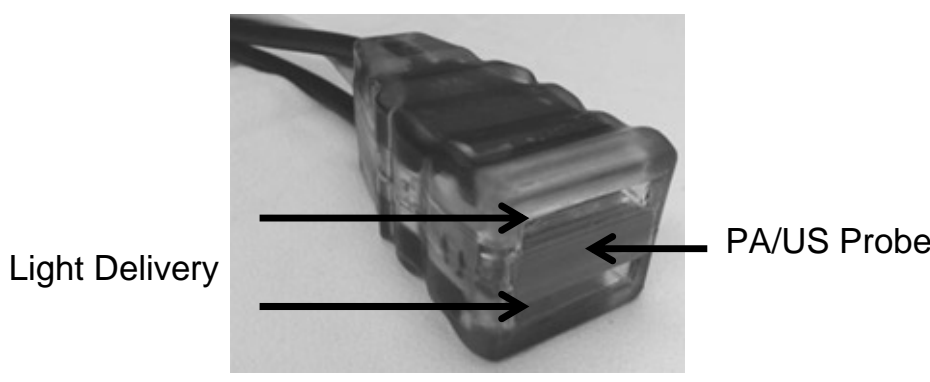

Figure 2. Hand-held PAT-US probe. The transducer features a flat surface for light delivery, and fiber bundles are integrated into the transducer housing for easier handling. 


\section{IN VIVO CLINICAL RESULTS}

Study participants received a single subcutaneous injection of methylene blue dye (5 mL, 2 $\mathrm{mg} / \mathrm{mL}$ ) with a 25 gauge needle near the areola in the same breast quadrant as the primary tumor. This technique was comparable to current methods utilized during routine SLNB. The breast was manually massaged for approximately 5 minutes to facilitate lymphatic flow to the axilla. PATUS imaging of the axilla was performed following methylene blue administration to identify the accumulation of methylene blue in suspected SLNs.

Figures 3a and 3b show corresponding US and PAT images, respectively, revealing a SLN. The co-registered PAT and US image (Figure 3c) shows the capability of the technology to locate lymph nodes via anatomical features in US, and to verify the lymph node as a sentinel node using PAT.

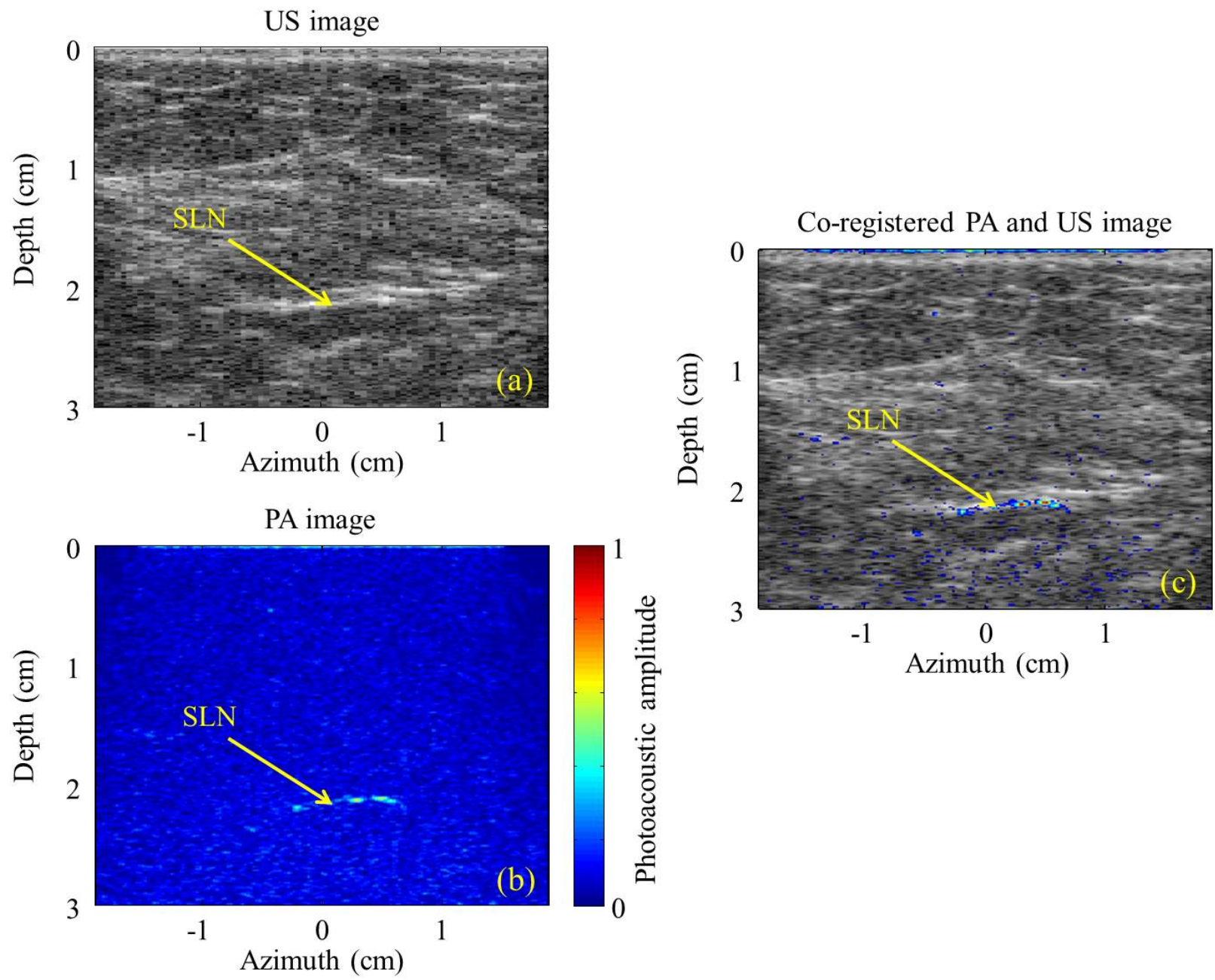

Figure 3. (a) In vivo US image of lymph node, (b) In vivo PAT image represented by optical absorption of methylene blue, (c) corresponding co-registered PAT-US image of the SLN. 


\section{CONCLUSIONS}

We successfully developed a dual-modality imaging system that integrates PAT and ultrasonography. Our preliminary results show that co-registered PAT and US imaging can detect SLNs in real-time. This approach offers significant benefits over the current standard of care; specifically, it would avoid the morbidity of the surgical SLNB technique and eliminate the need for radioactive isotopes in lymph node staging.

\section{ACKNOWLEDGEMENTS}

We thank Prof. James Ballard for his attentive reading of the manuscript. This work was sponsored by NIH grants R01 CA134539, DP1 EB016986 (NIH Director's Pioneer Award), R01 EB016963, and U54 CA136398. L. V. Wang has a financial interest in Endra, Inc., and Microphotoacoustics, Inc., which, however, did not support this work.

\section{REFERENCES}

[1] Krag D., Weaver D., Ashikaga T., Moffat F., Klimberg V. S., Shriver C., Feldman S., Kusminsky R., Gadd M., Kuhn J., Harlow S., and Beitsch P., "The sentinel node in breast cancer--a multicenter validation study,” New England Journal of Medicine 339, 941-946 (1998).

[2] McMasters K. M., Tuttle T. M., Carlson D. J., Brown C. M., Noyes R. D., Glaser R. L., Vennekotter D. J., Turk P. S., Tate P. S., Sardi A., Cerrito P. B., and Edwards M. J., "Sentinel lymph node biopsy for breast cancer: a suitable alternative to routine axillary dissection in multi-institutional practice when optimal technique is used," Journal of Clinical Oncology 18(13), 2560-2566 (2000).

[3] Singletary S. E., Allred C., Ashley P., Bassett L. W., Berry D., Bland K. I., Borgen P. I., Clark G., Edge S. B., Hayes D. F., Hughes L. L., Hutter R. V., Morrow M., Page D. L., Recht A., Theriault R. L., Thor A., Weaver D. L., Wieand H. S., and Greene F. L., "Revision of the American Joint Committee on Cancer staging system for breast cancer," Journal of Clinical Oncology 20, 3628-3636 (2002).

[4] Wang L. V. and Hu S., "Photoacoustic tomography: in vivo imaging from organelles to organs,” Science 335, 1458-1462 (2012).

[5] Erpelding T. N., Pramanik M., Kim C., Jankovic L., Maslov K., Guo Z., Margenthaler J. A., Pashley M. D., and Wang L. V., "Sentinel lymph nodes in the rat: noninvasive photoacoustic and US imaging with a clinical US system,” Radiology 256, 102-110 (2010).

[6] Kim C., Erpelding T. N., Jankovic L. and Wang L. V., "Performance benchmarks of an array-based hand-held photoacoustic probe adapted from a clinical ultrasound system for non-invasive sentinel lymph node imaging," Philosophical transactions. Series A, Mathematical, physical, and engineering sciences 369(1955), 4644-4650 (2011). 
[7] Kim C., Erpelding T. N., Jankovic L., Pashley M. D., and Wang L. V., "Deeply penetrating in vivo photoacoustic imaging using a clinical ultrasound array system," Biomedical Optics Express 1, 278-284 (2010).

[8] ANSI, American national standard for the safe use of lasers. ANSI z136.1-2007, Laser Institute of America, (2007). 